\title{
Analysis of Scientific Publications on Acinetobacter bacteremia in Web of Science
}

\author{
Sevil Alkan ${ }^{1}$ (D), Emine Kübra Dindar Demiray² (D), Emel Yıldız ${ }^{\mathbf{3}}$ (D), Can Özlü4 (D) \\ 1 Department of Infectious Diseases and Clinical Microbiology, Çanakkale Onsekiz Mart University School of Medicine, \\ Çanakkale, Turkey \\ 2 Infectious Disease Department, Bitlis State Hospital, Bitlis, Turkey \\ 3 Department of Anesthesia and Reanimation, Kütahya Health Sciences University Evliya Çelebi Training and Research \\ Hospital, Kütahya Health Sciences University School of Medicine, Kütahya, Turkey \\ 4 Department of Hematology, Kütahya Health Sciences University Evliya Çelebi Training and Research Hospital, Kütahya \\ Health Sciences University School of Medicine, Kütahya, Turkey
}

\begin{abstract}
Objective: We aimed to investigate the literature on Acinetobacter bacteremia (AB), which is always on the agenda because of increased mortality and morbidity.

Methods: We used the keywords "Acinetobacter and bacteremia" and limited our search to the journal articles. Retrieved data were analysed to present various bibliometric indicators while maps were visualised using the VOSviewer technique. Data pertaining to the growth of publications, the most active countries and institutions, the most cited journals, and the mapping of molecular mechanisms of resistance were analysed.

Results: A total of 179 journal articles were retrieved, with an average of 24.68 citations per article. The annual growth of $\mathrm{AB}$ articles showed an increasing pattern during the study period. The articles were usually written in English (96.08\%), and in 56.42\% the research area was infectious diseases (56.42\%). In terms of geography, three out of five countries were Asian in origin, and the leading country was Taiwan (30.72\%).

Conclusions: Publications on $A B$ have been increased in the last two decades. The most of the publications on $\mathrm{AB}$ contributed by Taiwan and the growing numbers in Asia and the globe. Keywords: Acinetobacter Bacteremia, Network Analysis, Scientific Publications, Web Of Science.
\end{abstract}

\section{INTRODUCTION}

A cinetobacter species are gaining resistance to antibiotics, and they became one of the most important pathogens causing healthcare-associated infections (HAI)

(1). It causes mortality up to $40 \%$, especially because of increased antibiotic resistance $(2,3)$. We aimed to investigate the publications in scientific journals on Acinetobacter bacteremia, which significant morbidity and mortality.

\section{MATERIALS AND METHOD}

a. Data source: This study was carried out to retrieve data from the journals indexed

\section{Corresponding Author: Sevil Alkan \\ E-mail: sevil3910@gmail.com}

Received: April 6, 2021 Accepted: April 28, 2021 Published: April 30, 2021

Suggested citation: Alkan S, Dindar Demiray EK, Yıldız E, Özlü C. Analysis of Scientific Publications on Acinetobacter bacteremia in Web of Science. Infect Dis Clin Microbiol 2021; 1: 39-44.

DOI: 10.36519/idem.2021.37 
in the Web of Science (WOS) database. Data were retrieved from the Science Citation Index-Expanded (SCI-E) of the WOS database (https://www.webofknowledge.com). Comprehensive bibliometric data and the SCI-EXPANDED, SSCI, A \& HCI, CPCI-S, CPCI-SSH, BKCI-S, BKCI-SSH, ESCI indexes database were retrieved from the Web of Science (WOS) Core Collection, which is considered as the optimum database for bibliometrics in previous studies (4-6).

\section{Search strategy and data collection}

The dataset from January 1970 to December 31, 2020, was obtained from the WOS Core Collection. The selected keywords used in the WOS database were those related keywords 'Acinetobacter' and 'bacteremia OR sepsis'. Language = English AND Document type = 'Article' were included.

The titles, years of publication, names of authors, nationalities, affiliations, keywords, names of publishing journals, abstracts of each record, and citations within the publications downloaded from WOS were saved as TXT files and imported into Microsoft Excel 2019. Çanakkale Onsekiz Mart University's online library and digital resources were used to access information.

\section{Bibliometric and visualised analysis}

The Hirsch (H) index and the impact factor (IF) of the publishing journals were used as indicators of the impact of publications (4-6).

\section{Analytical methods}

Retrieved data were analysed to present various bibliometric indicators while maps were visualised using the VOSviewer technique. VOSviewer (Leiden University, Leiden, Netherlands) is a software tool for the visualised analysis of the publications used

\section{HIGHLIGHTS}

- The reports about the Acinetobacter bacteremia have increased in last two decades.

- Emerging number of the reports on Acinetobacter bacteremia from Asia is significant.

- Diverse co-authorship and citation figures showed that this subject was a global concern.
Table 1. List of top ten active countries in publishing article on Acinetobacter bacteremia

\begin{tabular}{|l|c|c|c|}
\hline SCR & Country & Frequency & $\begin{array}{c}\text { Percentage of } \\
\text { total } \\
\text { publications } \\
(\mathrm{n}=179)\end{array}$ \\
\hline $1^{\text {st }}$ & Taiwan & 55 & 30.72 \\
\hline $2^{\text {th }}$ & United States of America & 28 & 15.64 \\
\hline $3^{\text {rd }}$ & South Korea & 20 & 11.17 \\
\hline $4^{\text {th }}$ & People's Rep. of China & 12 & 6.70 \\
\hline $5^{\text {th }}$ & Spain & 11 & 6.14 \\
\hline $6^{\text {th }}$ & Turkey & 9 & 5.02 \\
\hline $7^{\text {th }}$ & Thailand & 7 & 3.91 \\
\hline $8^{\text {th }}$ & Greece & 6 & 3.35 \\
\hline $9^{\text {th }}$ & Canada & 5 & 2.79 \\
\hline $9^{\text {th }}$ & France & 5 & 2.79 \\
\hline
\end{tabular}

SCR - standard competition ranking. Equal countries were given the same ranking number.

for bibliographic coupling, co-authorship, co-citation, and co-occurrence analysis (4-6).

\section{RESULTS}

\section{Analysis of global publications}

A total of 179 journal articles were retrieved, with an average of 24.68 citations per article. Even if the study includes the period between the years 1970 and 2020, the first article was in the year 1985.

The annual growth of Acinetobacter bacteremia articles showed an increasing pattern during the study period. Retrieved articles were written in three different languages, mainly English ( $\mathrm{n}=172 ; 96.08 \%)$ followed by Spanish $(n=5 ; 2.79 \%)$ and French $(n=2$; $1.11 \%)$.

The majority of the retrieved articles were from the research area of infectious disease $(\mathrm{n}=101 ; 56.42 \%)$, followed by microbiology ( $\mathrm{n}=73 ; 40.78 \%)$, immunology $(n=31 ; 17.31 \%)$, pharmacology $(n=24 ; 13.40 \%)$, medicine general internal $(n=20 ; 11.17 \%)$, public environmental occupation health $(\mathrm{n}=15 ; 8.38 \%)$. 


\section{Total Publications by Year}

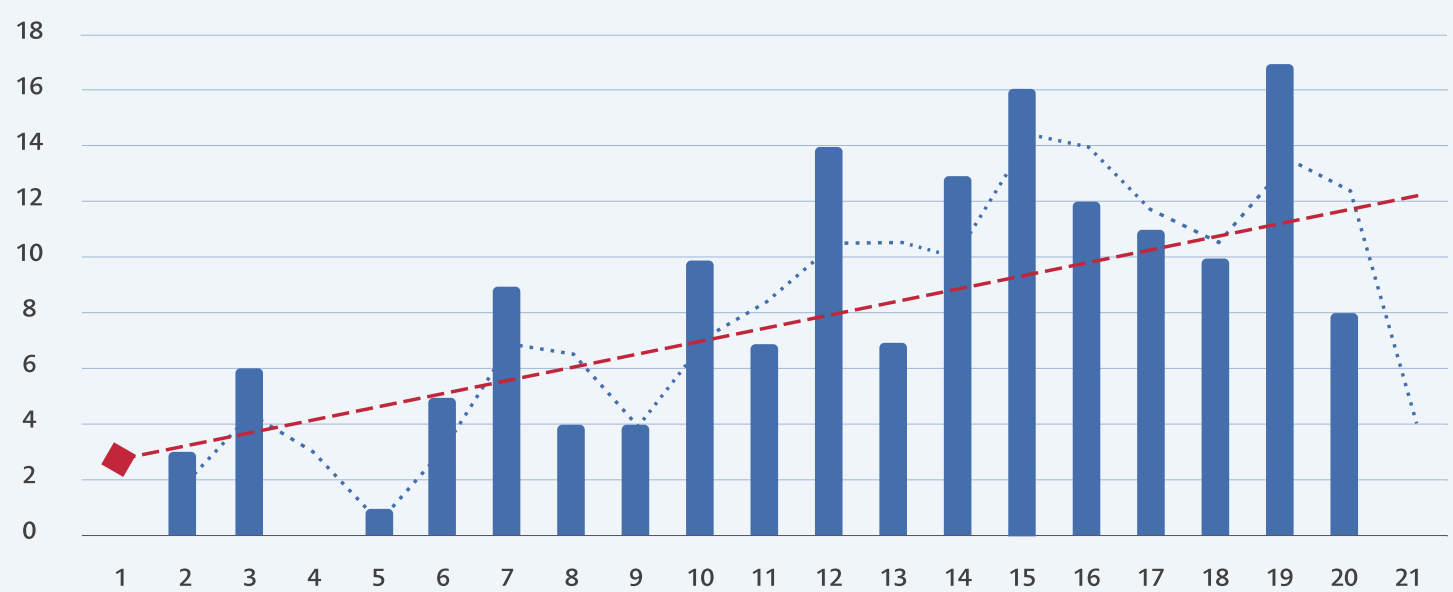

Figure 1. Graphics of total publication by years between 2001-2021. The blue dotted line expresses the median of the total value. The red dashed line expresses the trendline.

Sum of Times Cited per Year

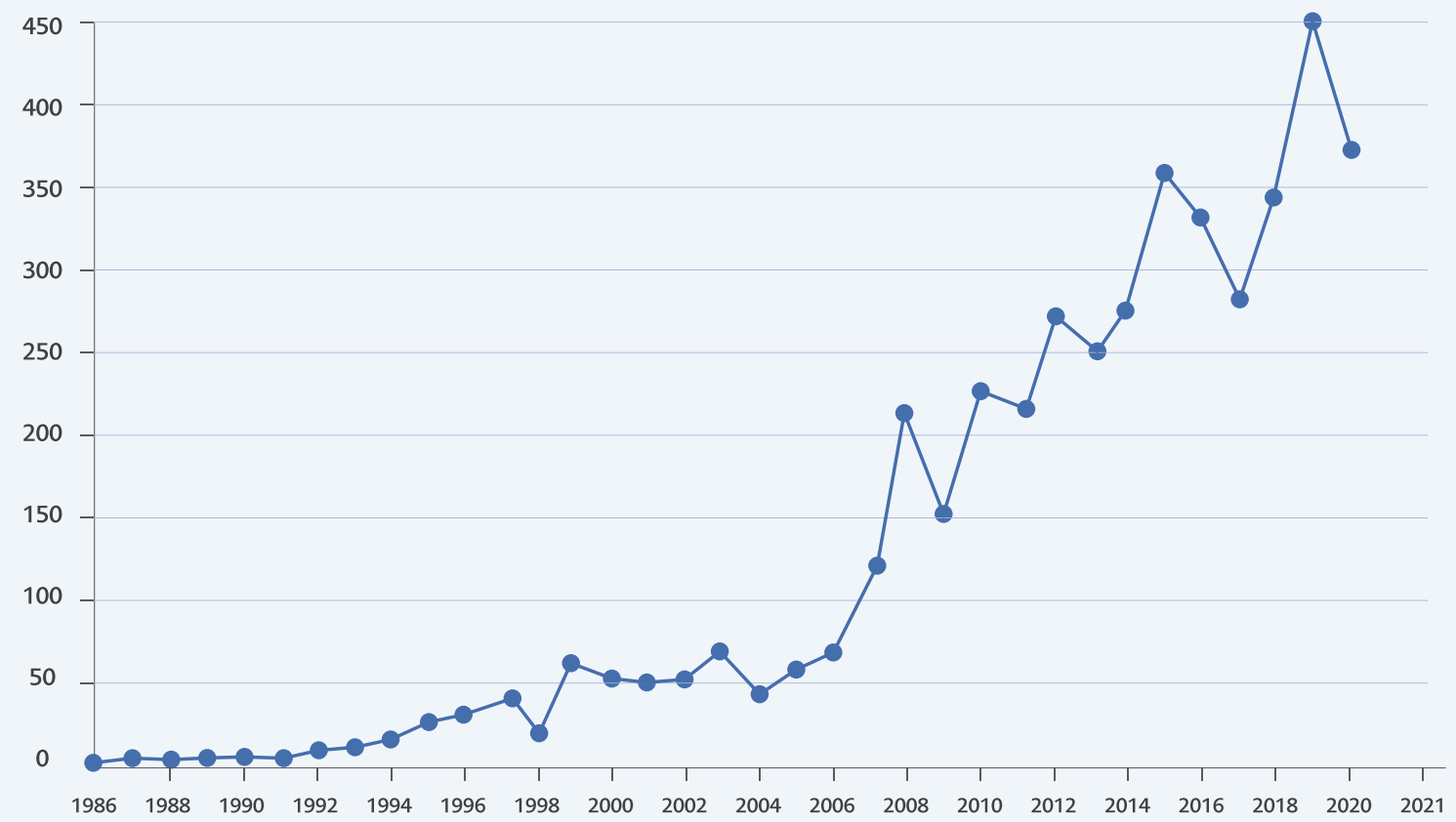

Figure 2. Graphics of citation by years between 1996-2021. The line refers to the citation number. 


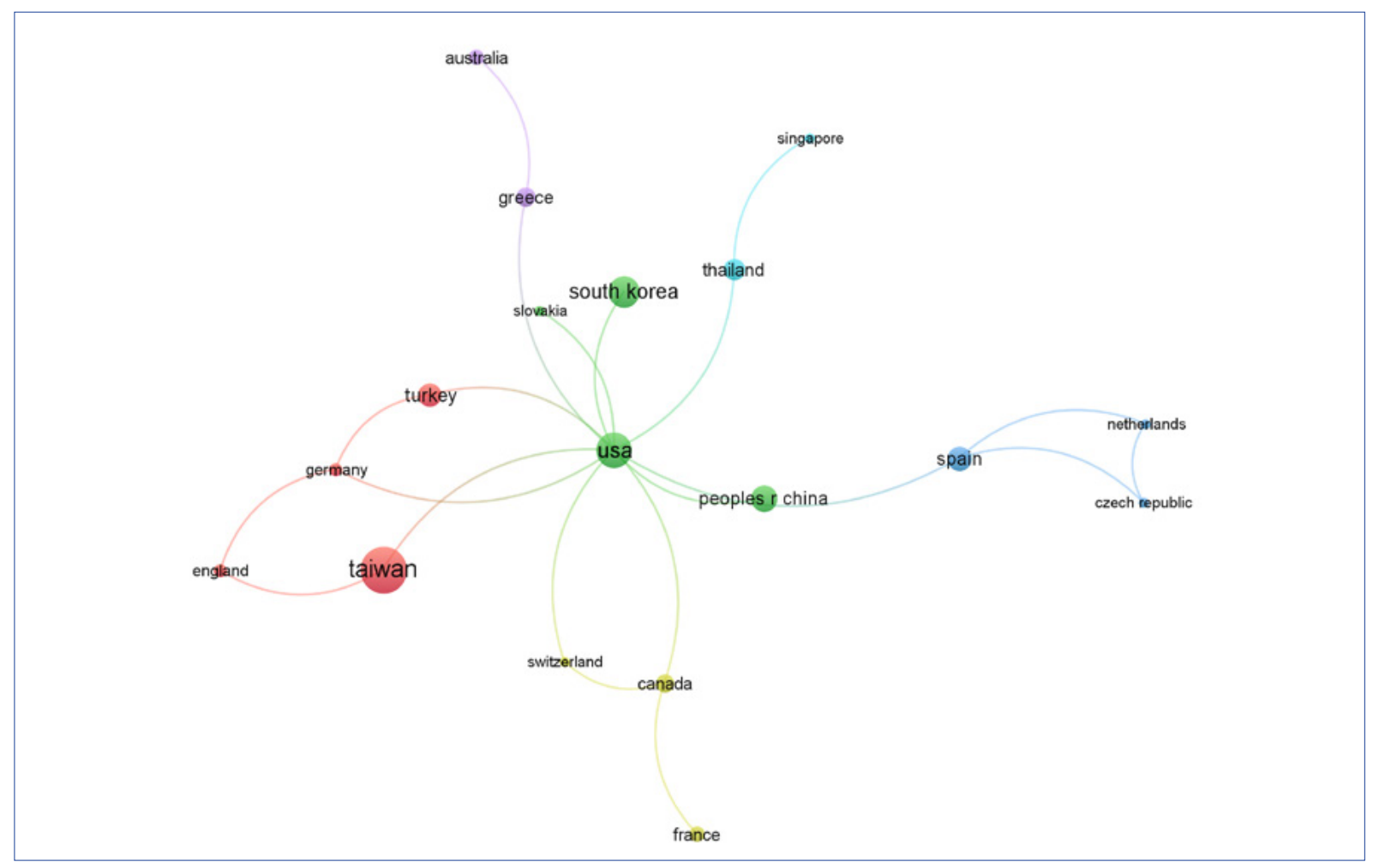

Figure3. Network visualisation map of co-authorship among countries with a minimum of one publication on Acinetobacter bacteremia. Lines connecting countries are indicative of collaboration. Thicker lines indicate stronger collaborations. Countries represented with larger circle size or font size had relatively more international collaboration.

\section{Active countries, institutions, and journals}

The leading country according to the number of publications was Taiwan $(\mathrm{n}=55 ; 30.72 \%)$, followed by USA ( $n=28 ; 15.64 \%)$, South Korea $(n=20 ; 11.17 \%)$, People's Republic of China ( $\mathrm{n}=12 ; 6.70 \%)$, Spain $(\mathrm{n}=11 ; 6.14 \%)$, Turkey $(\mathrm{n}=9 ; 5.02 \%)$. Other 27 countries around the globe were $24.61 \%(n=66)$. The list of active countries represents four different world regions: Northern and Southern America, Europe, Asia and Africa.

The articles were from 34 countries. The international collaboration which papers co-authored by authors from more than one country was classified as "international collaborations" carried out between 18 countries. These countries included Taiwan, the USA, Australia, England, Turkey, Germany, Greece, Slovakia, Switzerland, Canada, France, Spain, Netherlands, Czech Republic, South Korea, Thailand, Singapore, People's Republic of China. International collaboration analysis for active coun- tries, which must have at least one document, using the VOSviewer technique, showed 18 clusters of international collaboration (Figure 1).

\section{Citation numbers}

Retrieved articles received a total number of citations of 4417, an average of 24.68 citations per article. The H-index of the retrieved articles was 36. A total of 159 (88.82\%) were cited at least once, while the remaining $20(11.18 \%)$ articles were not cited at all. The top three cited articles on Acinetobacter bacteremia were published in Clinical Infectious Diseases (240), Medicine (188 citations) and American Journal of Epidemiology (150 citations).

\section{Growth of citations}

The annual citation growth of Acinetobacter bacteremia showed slow growth until the 2006s, followed by a dramatic increase in the last one and a half-decade. Figure 1 shows the annual growth of the citation on Acinetobacter bacteremia. The highest number of publications was seen in 2019, with a 


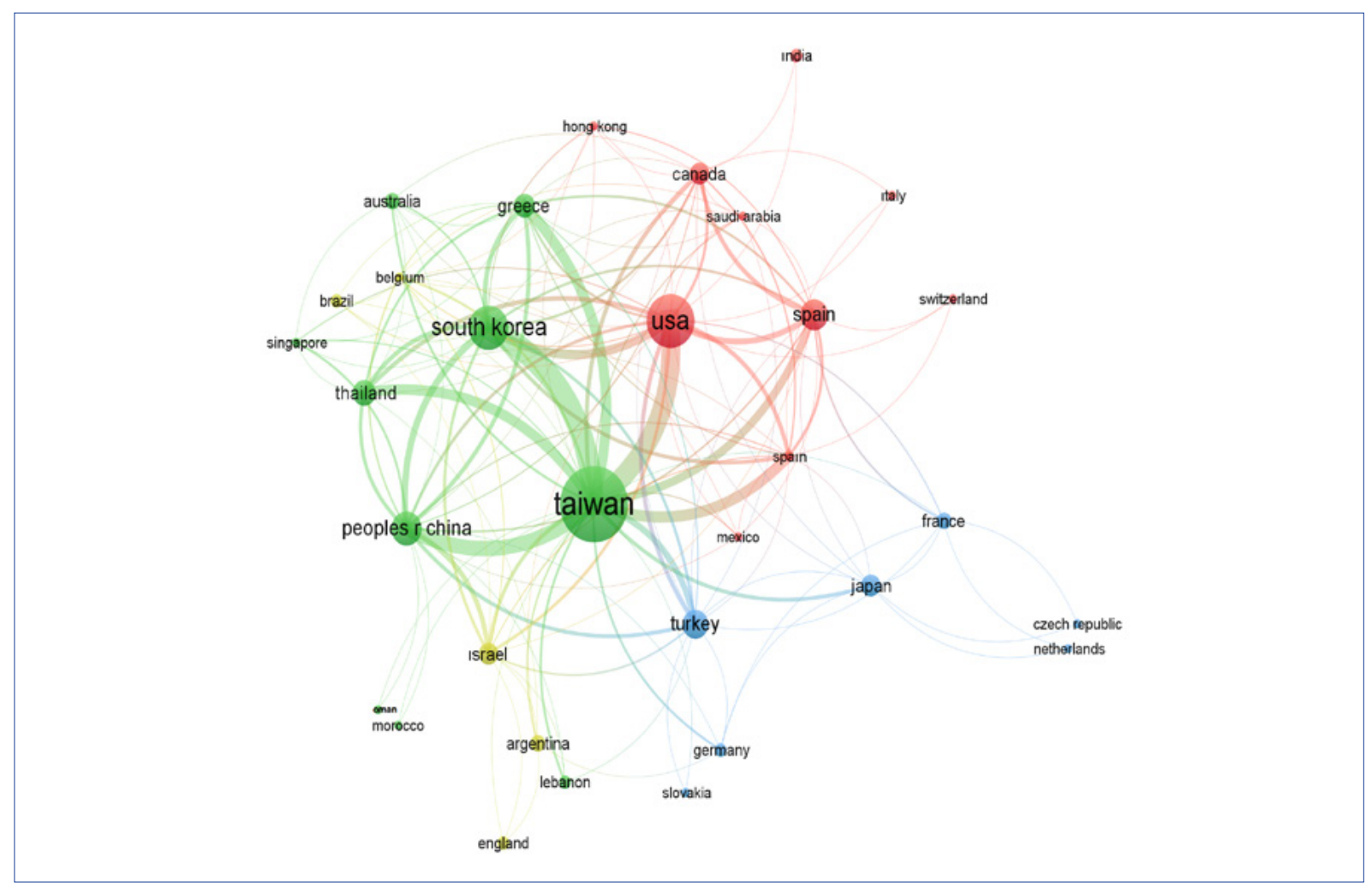

Figure 4. Network visualization map of citation map among countries with a minimum of one publication on Acinetobacter bacteremia. Lines connecting countries are indicative of collaboration. Thicker lines indicate stronger collaborations. Countries represented with larger circle size or font size had relatively more international collaboration.

total of 443 citations (Figure 2).

While the international countries were up above, the citation analysis of the 34 countries showed that the most (Figure 3,4).

\section{DISCUSSION}

We sought to give a bibliometric overview of the literature on Acinetobacter and bacteremia between 1970-2021, by using WOS database, which has been used in previously published bibliometric studies. We showed that publications Acinetobacter bacteremia have been increasing and growing rapidly in the past decade. Even with a low number of publications, the high H-index number indicates the importance of this subject to a variety of clinicians and researchers. Furthermore, the studies were from all over the world-this subject concerns almost all world regions. However, in the top five active countries, there were three Asian countries. Growing numbers of the study of Acinetobacter bacteremia in Asia was significant. The difference between co-authorship and citation figures showed that this subject is a global concern and needs many more international collaboration studies (co-authorship).

The current study has several limitations. The studies the in journals that were not indexed in the WOS database could not be included. Also, keywords were only in English, so publications written in other languages could have been missed. Another limitation was not including tther databases such as Scopus, Pubmed. Analyses were made only by the VOSviewer; other software such as CiteSpace II and Bibexcel) could be used in future studies.

In conclusion, publications on Acinetobacter bacteremia have been increased in recent two decades. Our study shows that the most of the publications on Acinetobacter bacteremia contributed by Taiwan, with a growing number of studies, especially in Asian countries. The rest of the world contributes a considerable share. This bibliometric analysis 
Ethical Approval: Not applicable. No approval is needed for bibliometric studies.

Peer-review: Externally peer-reviewed

Author Contributions: Concept - S.A.; Design - S.A., E.K.D.D.; Supervision S.A., E.K.D.D.; Materials - S.A., E.K.D.D.; Data Collection and/or Processing - S.A., E.K.D.D., E.Y., C.Ö.; Analysis and/or Interpretation - S.A., E.K.D.D., E.Y., C.Ö.; Literature Review - S.A., C.Ö.; Writer - S.A.; Critical Reviews - S.A., E.K.D.D., E.Y., C.Ö.

Conflict of Interest: The authors have no conflict of interest to declare.

Financial Disclosure: The authors declared that this study has received no financial support.

\section{REFERENCES}

1 Antunes LC, Visca P, Towner KJ. Acinetobacter baumannii: evolution of a global pathogen. Pathog Dis 2014; 71: 292- 301.

2 Chen CT, Wang YC, Kuo SC, Shih FH, Chen TL, How CK, et al. Community-acquired bloodstream infections caused by Acinetobacter baumannii: a matched case-control study. J Microbiol Immunol Infect 2018; 51: 629-35.

3 Liu Q, Li W, Du X, Li W, Zhong T, Tang Y, et al. Risk and prognostic factors for multidrug-resistant Acinetobacter baumannii complex bacteremia: a retrospective study in a tertiary hospital of West China. PLoS One 2015; 10: e0130701.
4 Xie L, Chen Z, Wang H, Zheng C, Jiang J. Bibliometric and visualized analysis of scientific publications on atlantoaxial spine surgery based on web of science and VOSviewer. World Neurosurg 2020;137: 435-42.e4.

5 Huang T, Wu H, Yang S, Su B, Tang K, Quan Z, et al. global trends of researches on sacral fracture surgery: a bibliometric study based on VOSviewer. Spine (Phila Pa 1976) 2020; 45: E721-E728.

6 Wang Y, Wang Q, Wei X, Shao J, Zhao J, Zhang Z, et al. Global scientific trends on exosome research during 2007-2016: a bibliometric analysis. Oncotarget 2017; 8: 48460-48470. 